\title{
Foreign Library Interface
}

\author{
by Daniel Adler
}

\begin{abstract}
We present an improved Foreign Function Interface (FFI) for R to call arbitary native functions without the need for $C$ wrapper code. Further we discuss a dynamic linkage framework for binding standard $C$ libraries to $\mathrm{R}$ across platforms using a universal type information format. The package rdyncall comprises the framework and an initial repository of crossplatform bindings for standard libraries such as (legacy and modern) OpenGL, the family of $S D L$ libraries and Expat. The package enables systemlevel programming using the R language; sample applications are given in the article. We outline the underlying automation tool-chain that extracts cross-platform bindings from $C$ headers, making the repository extendable and open for library developers.
\end{abstract}

\section{Introduction}

We present an improved Foreign Function Interface (FFI) for $\mathrm{R}$ that significantly reduces the amount of $\mathrm{C}$ wrapper code needed to interface with $\mathrm{C}$. We also introduce a dynamic linkage that binds the $C$ interface of a pre-compiled library (as a whole) to an interpreted programming environment such as $\mathrm{R}$ - hence the name Foreign Library Interface. Table 1 gives a list of the $C$ libraries currently supported across major R platforms. For each library supported, abstract interface specifications are declared in a compact platform-neutral text-based format stored in socalled DynPort file on a local repository.

$\mathrm{R}$ was choosen as the first language to implement a proof-of-concept implementation for this approach. This article describes the rdyncall package which implements a toolkit of low-level facilities that can be used as an alternative FFI to interface with C. It also facilitates direct and convenient access to common $C$ libraries from $\mathrm{R}$ without compilation.

The project was motivated by the fact that highquality software solutions implemented in portable $C$ are often not available in interpreter-based languages such as R. The pool of freely available C libraries is quite large and represents an invaluable resource for software development. For example, OpenGL (OpenGL Architecture Review Board et al., 2005) is the most portable and standard interface to accelerated graphics hardware for developing realtime graphics software. The combination of OpenGL with the Simple DirectMedia Layer (SDL) (Lantinga, 2009) core and extension libraries offers a foundation framework for developing interactive multime- dia applications that can run on a multitude of platforms.

\section{Foreign function interfaces}

FFIs provide the backbone of a language to interface with foreign code. Depending on the design of this service, it can largely unburden developers from writing additional wrapper code. In this section, we compare the built-in R FFI with that provided by rdyncall. We use a simple example that sketches the different work flow paths for making an $\mathrm{R}$ binding to a function from a foreign $\mathrm{C}$ library.

\section{FFI of base $\mathbf{R}$}

Suppose that we wish to invoke the $\mathrm{C}$ function sqrt of the Standard C Math library. The function is declared as follows in $\mathrm{C}$ :

double sqrt(double $\mathrm{x}$ );

The . C function from the base R FFI offers a call gate to $C$ code with very strict conversion rules, and strong limitations regarding argument- and returntypes: $R$ arguments are passed as $C$ pointers and $C$ return types are not supported, so only $C$ void functions, which are procedures, can be called. Given these limitations, we are not able to invoke the foreign sqrt function directly; intermediate wrapper code written in $C$ is needed:

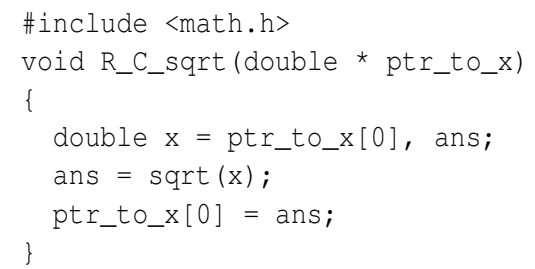

We assume that the wrapper code is deployed as a shared library in a package named testsqrt which links to the Standard C Math library ${ }^{1}$. Then we load the testsqrt package and call the $C$ wrapper function directly via . C.

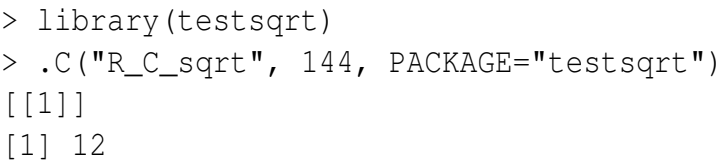

To make sqrt available as a public function, an additional $\mathrm{R}$ wrapper layer is needed to carry out type-safety checks:

\footnotetext{
${ }^{1}$ We omit here the details such as registering C functions which is described in detail in the R Manual 'Writing R Extensions' (R Development Core Team, 2010).
} 


\begin{tabular}{l|l|c|c|c}
\hline Lib/DynPort & Description & Functions & Constants & Struct/Union \\
\hline GL & OpenGL & 336 & 3254 & - \\
GLU & OpenGL Utility & 59 & 155 & - \\
R & R library & 238 & 700 & 27 \\
SDL & Audio/Video/UI abstraction & 201 & 416 & 34 \\
SDL_image & Pixel format loaders & 35 & 3 & - \\
SDL_mixer & Music format loaders and playing & 71 & 27 & - \\
SDL_net & Network programming & 34 & 5 & 3 \\
SDL_ttf & Font format loaders & 38 & 7 & - \\
cuda & GPU programming & 387 & 665 & 84 \\
expat & XML parsing framework & 65 & 70 & - \\
glew & GL extensions & 1857 & - & - \\
gl3 & OpenGL 3 (strict) & 317 & 838 & 1 \\
ode & Rigid Physics Engine & 547 & 109 & 11 \\
opencl & GPU programming & 79 & 263 & 10 \\
stdio & Standard I/O & 75 & 3 & - \\
\hline
\end{tabular}

Table 1: Overview of available DynPorts for portable C Libraries

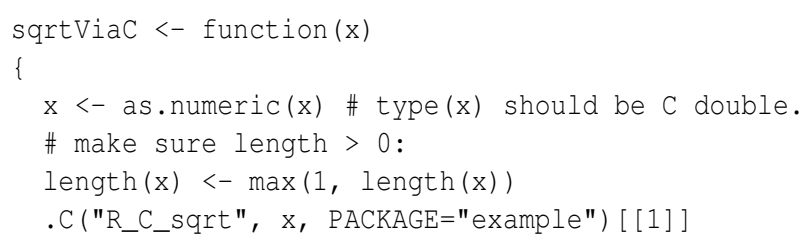

We can conclude that - in realistic settings - the built-in FFI of $\mathrm{R}$ almost always needs support by a wrapper layer written in C. The "foreign" in the FFI of base is in fact relegated to the $C$ wrapper layer.

\section{FFI of rdyncall}

rdyncall provides an alternative FFI for $\mathrm{R}$ that is accessible via the function . dyncall. In contrast to the base R FFI, which uses a C wrapper layer, the sqrt function is invoked dynamically and directly by the interpreter at run-time. Whereas the Standard C Math library was loaded implicitly via the testsqrt package, it now has to be loaded explicitly.

$\mathrm{R}$ offers functions to deal with shared libraries at run-time, but the location has to be specified as an absolute file path, which is platform-specific. A platform-portable solution is discussed in a following section on Portable loading of shared library. For now, we assume that the example is done on Mac OS $X$ where the Standard C Math library has the file path '/usr/lib/libm.dylib':

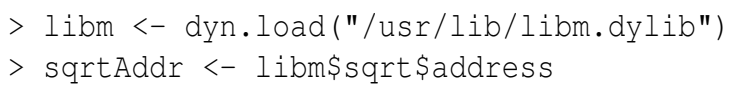

We first need to load the R package rdyncall:

\section{$>$ library (rdyncall)}

Finally, we invoke the foreign $C$ function sqrt $d i$ rectly via .dyncall:

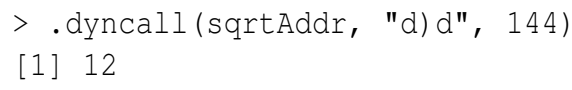

The last call pinpoints the core solution for a direct invocation of foreign code within $R$ : The first argument specifies the address of the foreign code, given as an external pointer. The second argument is a call signature that specifies the argument- and return types of the target $C$ function. This string "d) $d$ " specifies that the foreign function expects a double scalar argument and returns a double scalar value in accordance with the $C$ declaration of sqrt. Arguments following the call signature are passed to the foreign function in the form specified by the call signature. In the example we pass 144 as a $C$ double argument type as first argument and receive a $\mathrm{C}$ double value converted to an $\mathrm{R}$ numeric.

\section{Call signatures}

The introduction of a type descriptor for foreign functions is a key component that makes the FFI flexible and type-safe. The format of the call signature has the following pattern:

$$
\text { argument-types ' ) ' return-type }
$$

The signature can be derived from the $C$ function declaration: Argument types are specified first, in the direct left-to-right order of the corresponding $C$ function prototyp declaration, and are terminated by the symbol ' )' followed by a single return type signature.

Almost all fundamental $C$ types are supported and there is no restriction ${ }^{2}$ regarding the number of arguments supported to issue a call. Table 2 gives an

\footnotetext{
${ }^{2}$ The maximum number of arguments is limited by the amount of memory required for prebuffering a single call. It is currently fixed to 4 kilobyte (approx. 512-1024 arguments).
} 


\begin{tabular}{l|l|l|l}
\hline Type & Sign. & Type & Sign. \\
\hline void & $\mathrm{V}$ & bool & $\mathrm{B}$ \\
char & $\mathrm{C}$ & unsigned char & $\mathrm{C}$ \\
short & $\mathrm{S}$ & unsigned short & $\mathrm{S}$ \\
int & $\mathrm{I}$ & unsigned int & $\mathrm{I}$ \\
long & $\mathrm{J}$ & unsigned long & $\mathrm{J}$ \\
long long & $\mathrm{I}$ & unsigned long long & $\mathrm{L}$ \\
float & $\mathrm{f}$ & double & $\mathrm{d}$ \\
void* & $\mathrm{p}$ & struct name & $\star<$ name $>$ \\
type* & $\star \ldots$ & const char* & $\mathrm{Z}$ \\
\hline
\end{tabular}

Table 2: C/C++ Types and Signatures

\begin{tabular}{ll|l}
\hline C function declaration & Call signature \\
\hline void & rsort_with_index(double*,int*,int n) & $* d^{*} i$ ) v \\
SDL_Surface * SDL_SetVideoMode(int,int,int,Uint32_t) & iiil)*<SDL_Surface> \\
void & glClear(GLfloat,GLfloat, GLfloat, GLfloat) & ffff)v \\
\hline
\end{tabular}

Table 3: Examples of $C$ functions and corresponding call signatures

overview of supported $C$ types and the corresponding text encoding; Table 3 provides some examples of $C$ functions and call signatures.

A public $\mathrm{R}$ function that encapsulates the details of the sqrt call is simply defined by

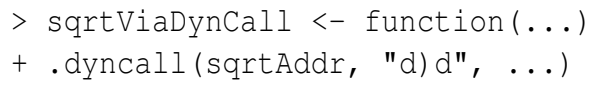

No further guard code is needed here because .dyncall has built-in type checks that are specified by the signature. In contrast to the $\mathrm{R}$ wrapper code using . C, no explicit cast of the arguments via as numeric is required, because automatic coercion rules for fundamental types are implemented as specified by the call signature. For example, using the integer literal $144 \mathrm{~L}$ instead of double works here as well.

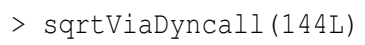

If any incompatibility is detected, such as a wrong number of arguments, empty atomic vectors or incompatible type mappings, the invocation is aborted and an error is reported without risking an application crash.

Pointer type arguments, expressed via 'p', are handled differently. The type signature ' $\mathrm{p}$ ' indicates that the argument is an address. When passing $\mathrm{R}$ atomic vectors, the $\mathrm{C}$ argument value is the address of the first element of the vector. External pointers and the NULL object can also be passed as values for pointer type arguments. Automatic coercion is deliberately not implemented for pointer types. This is to support $C$ functions that write into memory referenced by out pointer types.
Typed pointers, specified by the prefix ' $*$ ' followed by the signature of the base type, offer a measure of type-safety for pointer types; if an $R$ vector is passed and the $\mathrm{R}$ atomic type does not match the base type, the call will be rejected. Typed pointers to $C$ struct and union types are also supported; they are briefly described in the section Handling of C Types in $R$.

In contrast to the R FFI, where the argument conversion is dictated solely by the $\mathrm{R}$ argument type at call-time in a one-way fashion, the introduction of an additional specification with a call signature gives several advantages.

- Almost all possible $C$ functions can be invoked by a single interface; no additional $C$ wrapper is required.

- The built-in type-safety checks enhance stability and significantly reduce the need for assertion code.

- The same call signature works across platforms, given that the $\mathrm{C}$ function type remains constant.

- Given that our FFI is implemented in multiple languages (e.g. Python, Ruby, Perl, Lua), call signatures represent a universal type description for $\mathrm{C}$ libraries.

\section{Package overview}

Besides dynamic calling of foreign code, the package provides essential facilities for interoperability between the $\mathrm{R}$ and $\mathrm{C}$ programming languages. An 


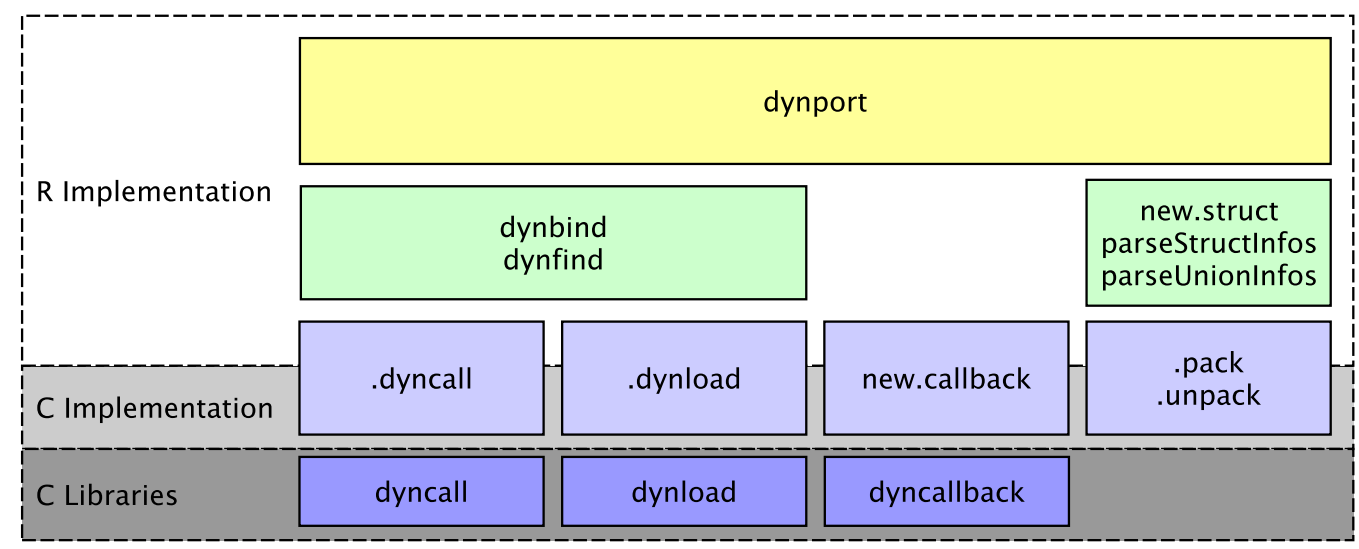

Figure 1: Package Overview

overview of components that make up the package is given in Figure 1.

We already described the .dyncall FFI. It is followed by a brief description of portable loading of shared libraries using dynfind, installation of wrappers via dynbind, handling of foreign data types via new. struct and wrapping of $R$ functions as $C$ callbacks via new.callback. Finally the high-level dynport interface for accessing whole $\mathrm{C}$ libraries is briefly discussed. The technical details at low-level of some components are described briefly in the section Architecture.

\section{Portable loading of shared libraries}

The portable loading of shared libraries across platforms is not trivial because the file path is different across operating systems. Referring back to the previous example, to load a particular library in a portable fashion, one would have to check the platform to locate the C library. ${ }^{3}$

Although there is variation among the operating systems, library file paths and search patterns have common structures. For example, among all the different locations, prefixes and suffixes, there is a part within a full library filename that can be taken as a short library name or label.

The function dynfind takes a list of short library names to locate a library using common search heuristics. For example, to load the Standard C Math library, depending on the operating system the library is either the Microsoft Visual C Run-Time DLL labeled 'msvcrt' on Windows or the Standard C Math shared library labeled ' $m$ ' or ' $c$ ' on other operating systems.

$>$ mLib <- dynfind(c("msvert", "m","c"))

dynfind also supports more exotic schemes, such as Mac OS X Framework folders. Depending on the library, it is sometimes enough to have a single short filename - e.g. "expat" for the Expat library.

\section{Wrapping C libraries}

Functional $\mathrm{R}$ interfaces to foreign code can be defined with small $\mathrm{R}$ wrapper functions, which effectively delegate to .dyncall. Each function interface is parameterized by a target address and a matching call signature.

f $<-$ function (...) . dyncall (target, signature, ...)

Since an Application Programming Interface (API) often consist of hundreds of functions (see Table 1), dynbind can create and install a batch of function wrappers for a library with a single call by using a $l i$ brary signature that consists of concatenated function names and signatures separated by semicolons.

For example, to install wrappers to the $C$ functions sqrt, sin and cos from the math library, one could use

$>$ dynbind( c("msvert", "m", "c"),

+ "sqrt $(d) d ; \sin (d) d) ; \cos (d) d ; "$ )

The function call has the side-effect that three $\mathrm{R}$ wrapper functions are created and stored in an environment that defaults to the global environment. Let us review the sin wrapper (on the 64-bit Version of $\mathrm{R}$ running on Mac OS X 10.6):

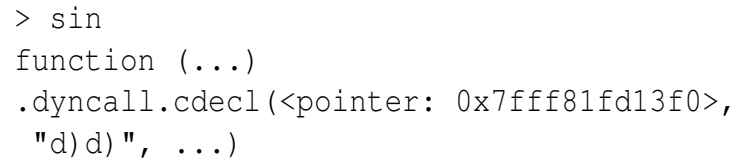

The wrapper directly uses the address of the sin symbol from the Standard C Math library. In addition, the wrapper uses .dyncall.cdecl, which is a concrete selector of a particular calling convention, as outlined below.

\footnotetext{
${ }^{3}$ Possible C math library names are 'libm.so' and 'MSVCRT.DLL' in locations such as '/lib', '/usr/lib', '/lib64', '/usr/lib64', 'C:IWINDOWSISYSTEM32' etc..
} 


\section{Calling conventions}

Calling conventions specify how arguments and return values are passed across sub-routines and functions at machine-level. This information is vital for interfacing with the binary interface of $\mathrm{C}$ libraries. The package has support for multiple calling conventions. Calling conventions are controlled by .dyncall via the named argument callmode to specify a non-default calling convention. Most supported operating systems and platforms only have support for a single "default" calling convention at run-time. An exception to this is the Microsoft Windows platform on the Intel $i 386$ processor architecture: While the default $\mathrm{C}$ calling convention on $i 386$ (excluding Plan9) is "default", system shared libraries from Microsoft such as 'KERNEL32.DLL', 'USER32.DLL' as well as the OpenGL library 'OPENGL32.DLL' use the "stdcall" calling convention. Only on this platform does the callmode argument have an effect. All other platforms currently ignore this argument.

\section{Handling of $\mathbf{C}$ types in $\mathbf{R}$}

C APIs often make use of high-level C struct and union types for exchanging information. Thus, to make interoperability work at that level the handling of $C$ data types is addressed by the package.

To illustrate this concept we consider the following example: A user-interface library has a function to set the $2 \mathrm{D}$ coordinates and dimension of a graphical output window. The coordinates are specified using a $C$ struct Rect data type and the $C$ function receives a pointer to that object:

void setWindowRect (struct Rect *pRect);

The structure type is defined as follows:

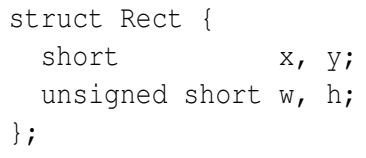

Before we can issue a call, we have to allocate an object of that size and initialize the fields with values encoded in $C$ types that are not part of the supported set of $\mathrm{R}$ data types. The framework provides $R$ helper functions and objects to deal with $C$ data types. Type information objects can be created with a description of the $C$ structure type. First, we create a type information object in $\mathrm{R}$ for the struct Rect $\mathrm{C}$ data type with the function parseStruct Infos using a structure type signature.

$>$ parseStructInfos ("Rect $\{$ SSSS $\}$ x y w h;")

After registration, an $\mathrm{R}$ object named Rect is installed that contains $\mathrm{C}$ type information that corresponds to struct Rect. The format of a structure type signature has the following pattern:

\section{Struct-name ' \{' Field-types ' $\}$ ' Field-names '; '}

Field-types use the same type signature encoding as that of call signatures for argument and return types (Table 2). Field-names consist of a list of whitespace separated names, that label each field component left to right.

An instance of a $C$ type can be allocated via new.struct:

\section{$>r<-$ new.struct (Rect)}

Finally, the extraction ('\$', '[') and replacement('\$<-', '[<-') operators can be used to access structure fields symbolically. During value transfer between $\mathrm{R}$ and $\mathrm{C}$, automatic conversion of values with respect to the underlying $C$ field type takes place.

$>r \$ \mathrm{x}<--10 ; r \$ \mathrm{y}<--20 ; r \$ \mathrm{w}<-40 ; r \$ \mathrm{~h}<-30$

In this example, $\mathrm{R}$ numeric values are converted on the fly to signed and unsigned short integers (usually 16-bit values). On printing $r$ a detailed picture of the data object is given:

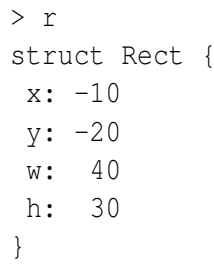

At low-level, one can see that $r$ is stored as an $\mathrm{R}$ raw vector object:

$>r[]$

[1] f6 ff ec ff 2800 le 00

attr (, "struct")

[1] "Rect"

To follow the example, we issue a foreign function call to setRect via .dyncall and pass in the $r$ object, assuming the library is loaded and the symbol is resolved and stored in an external pointer object named setWindowRectAddr:

$>$.dyncall ( setWindowRectAddr, "*<Rect $>$ ) v", r)

We make use of a typed pointer expression $1 *<$ Rect $>$ ' instead of the untyped pointer signature ' $p$ ', which would also work but does not prevent users from passing other objects that do not reference a struct Rect data object. Typed pointer expressions increase type-safety and use the pattern $1 *<$ Type-Name $>1$. The invocation will be rejected if the argument passed in is not of $C$ type Rect. As $r$ is tagged with an attribute struct that refers to Rect, the call will be issued. Typed pointers can also occur as return types that permit the manipulation of returned objects in the same symbolic manner as above.

C union types are supported as well but use the parseUnionInfos function instead for registration, and a slightly different signature format: 


\section{Union-name '। ' Field-types ' \} ' Field-names ' ; '}

The underlying low-level $C$ type read and write operations and conversions from $\mathrm{R}$ data types are performed by the functions .pack and .unpack. These can be used for various low-level operations as well, such as dereferencing of pointer to pointers.

$\mathrm{R}$ objects such as external pointers and atomic raw, integer and numeric vectors can be used as $C$ struct/union types via the attribute struct. To cast a type in the style of $C$, one can use as struct.

\section{Wrapping $\mathbf{R}$ functions as $\mathbf{C}$ callbacks}

Some C libraries, such as user-interface toolkits and I/O processing frameworks, use callbacks as part of their interface to enable registration and activation of user-supplied event handlers. A callback is a userdefined function that has a library-defined function type. Callbacks are usually registered via a registration function offered by the library interface and are activated later from within a library run-time context.

rdyncall has support for wrapping ordinary $\mathrm{R}$ functions as $\mathrm{C}$ callbacks via the function new. callback. Callback wrappers are defined by a callback signature and the user-supplied $\mathrm{R}$ function to be wrapped. Callback signatures look very similar to call signatures and should match the functional type of the underlying $C$ callback. new. callback returns an external pointer that can be used as a low-level function pointer for the registration as a $\mathrm{C}$ callback. See Section Parsing XML using Expat below for applications of new.callback.

\section{Foreign library interface}

At the highest level, rdyncall provides the front-end function dynport to dynamically set up an interface to a C Application Programming Interface. This includes loading of the corresponding shared C library and resolving of symbols. During the binding process, a new $\mathrm{R}$ name space (Tierney, 2003) will be populated with thin $\mathrm{R}$ wrapper objects that represent abstractions to $C$ counterparts such as functions, pointers-to-functions, type-information objects for $C$ struct and union types and symbolic constant equivalents of $C$ enums and macro definitions. The mechanism works across platforms; as long as the corresponding shared libraries of a DynPort have been installed in a system standard location on the host.

An initial repository of DynPorts is available in the package that provides bindings for several popular C APIs; see Table 1 for available bindings.

\section{Sample applications}

We give examples that demonstrate the direct usage of $\mathrm{C}$ APIs from within $\mathrm{R}$ through the rdyncall pack- age. The $\mathrm{R}$ interface to $\mathrm{C}$ libraries looks very similar to the actual C API. For details on the usage of a particular $C$ library, the programming manuals and documentation of the libraries should be consulted.

Before loading $\mathrm{R}$ bindings via dynport, the shared library should have been installed onto the system. Currently this is to be done manually and the installation method depends on the target operating system. While OpenGL and Expat is often pre-installed on typical desktop-systems, SDL usually has to be installed explicitly which is described in the package; see ?' rdyncall-demos ' for details.

\section{OpenGL programming in $R$}

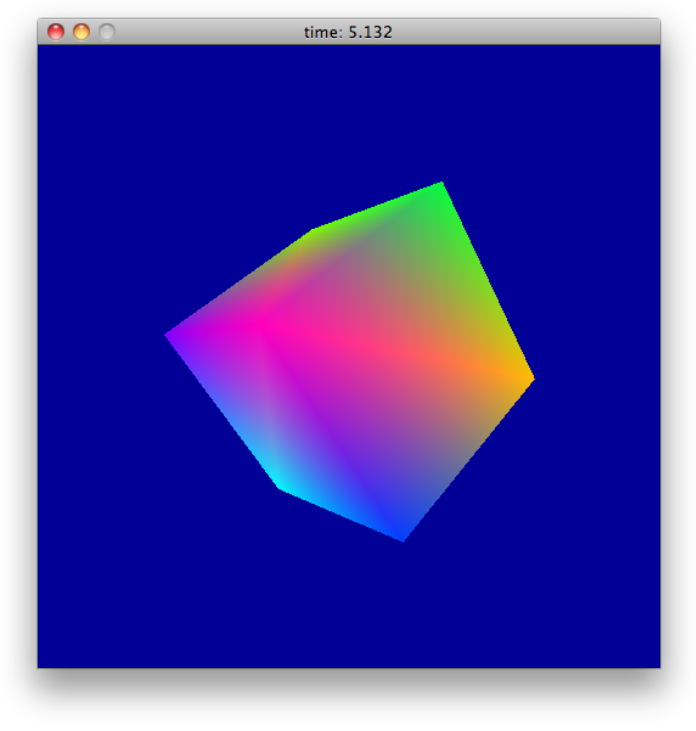

Figure 2: demo (SDL)

In the first example, we make use of the Simple DirectMedia Layer library (SDL) (Pendleton, 2003) and the Open Graphics Library (OpenGL) (OpenGL Architecture Review Board et al., 2005) to implement a portable multimedia application skeleton in R.

We first need to load bindings to SDL and OpenGL via dynport:

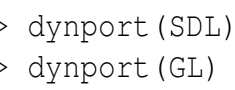

Now we initialize the SDL library, e.g. we initialize the video subsystem, and open a $640 \times 480$ window surface in 32-bit color depths with support for OpenGL rendering:

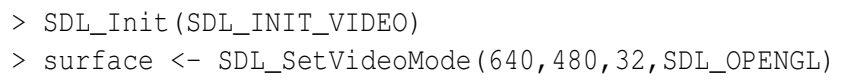

Next, we implement the application loop which updates the display repeatedly and processes the event queue until a quit request is issued by the user via the window close button. 


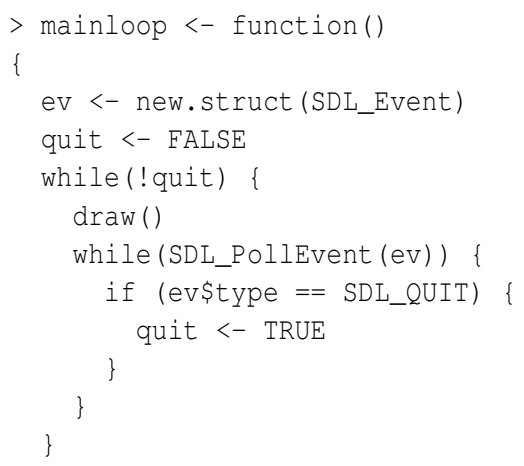

SDL event processing is implemented by collecting events that occur in a queue. Typical SDL applications poll the event queue once per update frame by calling SDL_PollEvent with a pointer to a userallocated buffer of $C$ type union SDL_Event. Event records have a common type identifier which is set to SDL_QUIT when a quit event has occurred, e.g. when users press a close button on a window.

Next we implement our draw function making use of the OpenGL API. We clear the background with a blue color and draw a light-green rectangle.

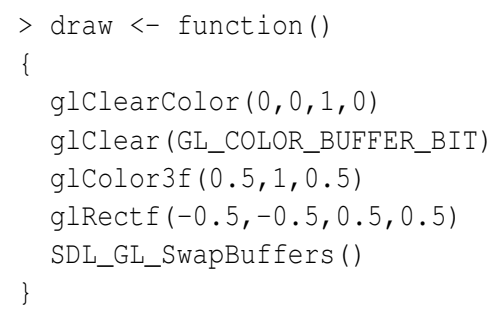

Now we can run the application mainloop.

\section{$>$ mainloop()}

To stop the application, we press the close button of the window. A similar example is also available via demo (SDL). Here the draw function displays a rotating 3D cube displayed in Figure 2.

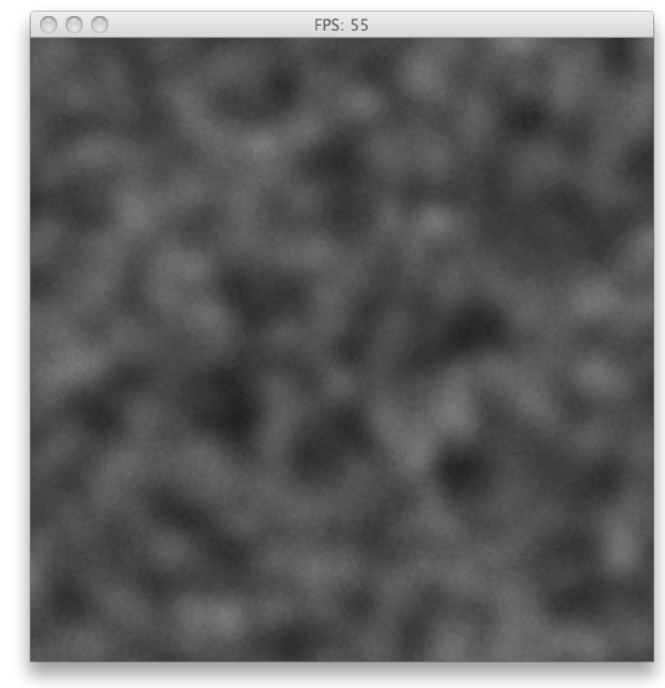

Figure 3: demo (randomfield) demo (randomfield) gives a slightly more scientific application of OpenGL and R: Random fields of $512 \times 512$ size are generated via blending of 5000 texture mapped 2D gaussian kernels. The counter in the window title bar gives the number of matrices generated per second (see Figure 3 ). When clicking on the animation window, the current frame and matrix is passed to $\mathrm{R}$ and plotted. While several dozens of matrices are computed and drawn per second using OpenGL, it takes several seconds to plot a single matrix in $\mathrm{R}$ using image () .

\section{Parsing XML using Expat}

In the second example, we use the Expat XML Parser library (Clark, 2007; Kim, 2001) to implement a stream-oriented XML parser suitable for very large documents. In Expat, custom XML parsers are implemented by defining functions that are registered as callbacks to be invoked on events that occur during parsing, such as the start and end of XML tags. In our second example, we create a simple parser skeleton that prints the start and end tag names.

First we load R bindings for Expat via dynport.

$>\operatorname{dynport}($ expat)

Next we create an abstract parser object via the C function XML_ParserCreate that receives one argument of type $C$ string to specify a desired character encoding that overrides the document encoding declaration. We want to pass a null pointer (NULL) here. In the .dyncall FFI C null pointer values for pointer types are expressed via the R NULL value:

$>\mathrm{p}<-\mathrm{XML}$ _ParserCreate (NULL)

The $C$ interface for registering start- and end-tag event handler callbacks is given below:

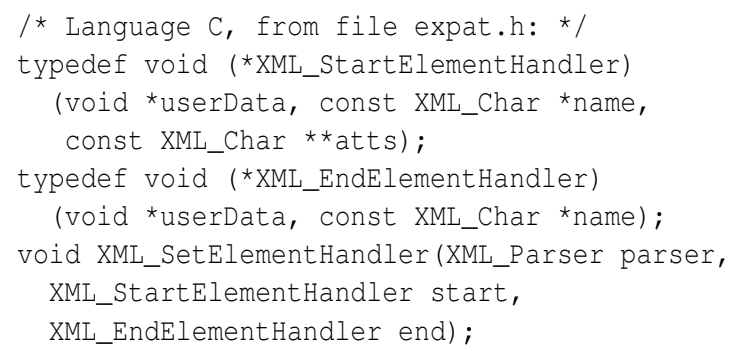

We implement the callbacks as $\mathrm{R}$ functions that print the event and tag name. They are wrapped as $C$ callback pointers via new. cal lback using a matching callback signature. The second argument name of type C string in both callbacks, XML_StartElementHandler and XML_EndElementHandler, is of primary interest in this example; this argument passes over the XML tag name. $C$ strings are handled in a special way by the .dyncall FFI because they have to be copied as $\mathrm{R}$ character objects. The special type signature ' $\mathrm{Z}$ ' is used to denote a $C$ string type. The other arguments are simply denoted as untyped pointers using ' $\mathrm{p}$ ': 


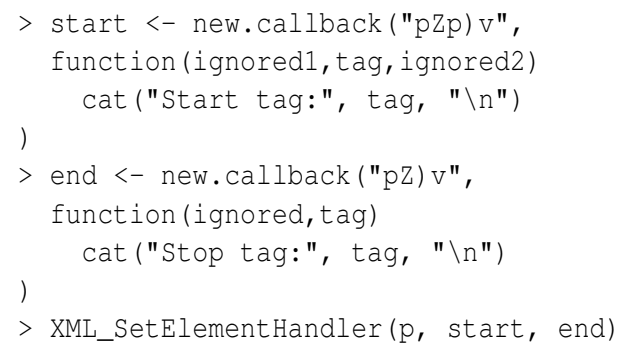

To test the parser we create a sample document stored in a character object named text and pass it to the parse function XML_Parse:

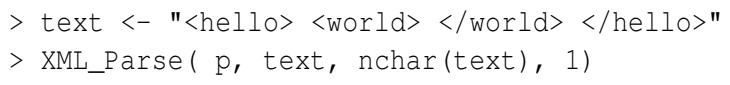

The resulting output is

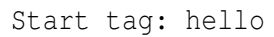

Expat supports processing of very large XML documents in a chunk-based manner by calling XML_Parse several times, where the last argument is used as indicator for the final chunk of the document.

\section{Architecture}

The core implementation of the FFI, callback wrapping and loading of code is based on small C libraries of the DynCall project (Adler and Philipp, 2011).

The implementation of the FFI is based on the dyncall $C$ library, which provides an abstraction for making arbitrary machine-level calls offering a universal C interface for scripting language interpreters. It has support for almost all fundamental $\mathrm{C}$ argument/return types ${ }^{4}$ and multiple calling conventions, and is open for extension to other platforms and binary standards. Generic call implementations for the following processor architectures are supported: Intel i386 32-bit, AMD 64-bit, PowerPC 32bit, ARM (including Thumb extension), MIPS 32/64bit and SPARC 32/64-bit including support for several platform-, processor- and compiler-specific calling conventions.

The dyncallback C library implements generic callback handling. Callback handlers receive calls from $\mathrm{C}$ and they forward the call, including conversion of arguments, to a function of a scriptinglanguage interpreter. A subset of architectures from the above is currently supported here: i386, AMD64 and ARM, and partial support for PowerPC 32-bit on Mac OS X/Darwin.

Besides the processor architecture, the libraries support various operating systems such as Linux, Mac OS X, Windows, the BSD family, Solaris, Haiku,
Minix and Plan9. Support for embedded platforms such as Playstation Portable, Nintendo DS and iOS is available as well. FFI implementations for other languages such as Python (van Rossum and Drake, Jr., 2005), Lua (Ierusalimschy et al., 1996) and Ruby (Flanagan and Matsumoto, 2008) are available from the DynCall project source repository.

The source tree supports various build tools such as gcc, msvc, SunPro, pcc, llvm and supports several make tools (BSD,C,GNU,N,Sun). A common abstraction layer for assembler dialects helps to develop cross-operating system call kernel. Due to the generic implementation and simple design, the libraries are quite small (the dyncall library for Mac OS X/AMD64 is $24 \mathrm{~kb}$ ).

To test stability of the libraries, a suite of testing frameworks is available, including test-case generators with support for structured or random case studies and for testing extreme scenarios with large number of arguments. Prior to each release, the libraries and tests are built for a large set of architectures on DynOS (Philipp, 2011); a batch-build system using CPU emulators such as QEmu (Bellard, 2005) and GXEmul (Gavare, 2010), and various operating system images to test the release candidates and to create pre-built binary releases of the library.

\section{Creation of DynPort files}

The creation of DynPort files from $C$ header files is briefly described next. A tool chain, comprising of freely available components, is applied once on a build machine as depicted in Figure 4.

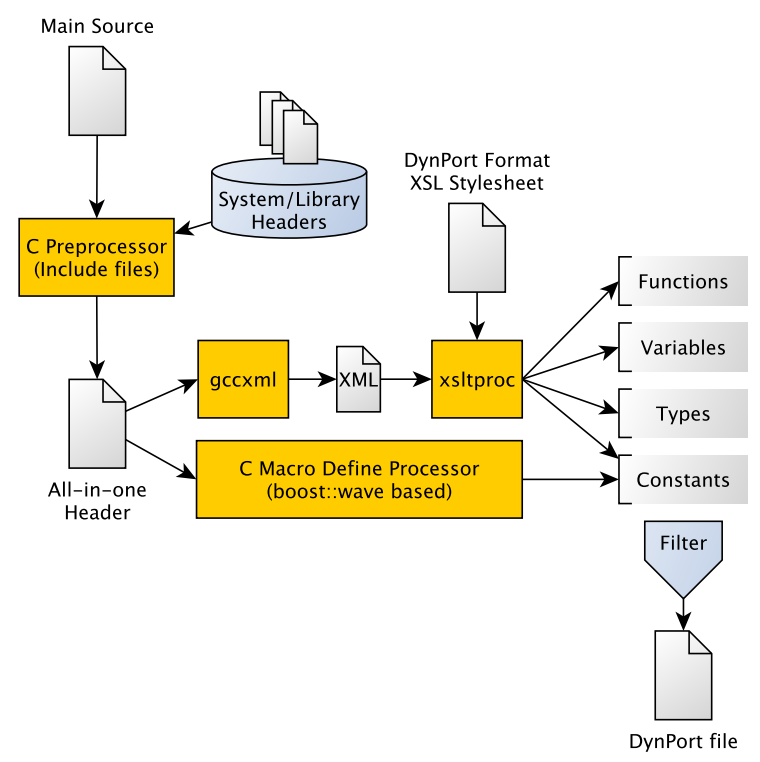

Figure 4: Tool-chain to create DynPort files from C headers

At first a main source file references the $C$ header files of the library that should be made accessable via

\footnotetext{
${ }^{4}$ Passing of long double, struct and union argument/return $\mathrm{C}$ value types are currently work in progress.
} 
dynport. In a preprocessing phase the GNU C Macro Processor is used to process all \#include statements using standard system search paths to create a concatenated All-In-One source file. GCC-XML (King, 2004), a modified version of the GNU C compiler, transforms $C$ header declarations to XML. The XML is further transformed to the final type signature format using xslproc (Veillard and Reese, 2009), a XSLT (Clark, 2001) processor, and a custom XSL stylesheet that has been implemented for the actual transformation from GCC-XML to the type signature text format.

C Macro \#define statements are handled separately by a custom $C$ Preprocessor implemented in $\mathrm{C}++$ using the boost wave library (Kaiser, 2011). An optional filter stage is used to include only elements with a certain pattern, such as a common prefix usually found in many libraries, e.g. 'SDL_'. In a last step, the various fragments are assembled into a single text-file that represents the DynPort file.

\section{Limitations}

During the creation of DynPort files, we encountered some cases (mainly for the SDL library) where we had to comment out some symbolic assignments (derived from $\mathrm{C}$ macro definitions) manually. These could not be converted as-is into valid $R$ assignments because they consist of complex $C$ expressions such as bit-shift operations. One could solve this problem by integrating a $\mathrm{C}$ interpreter within the tool-chain that deduces the appropriate type and value information from the replacement part of each $C$ macro definitions; definitions with incomplete type could be rejected and constant values could be stored in a language-neutral encoding.

In order to use a single DynPort for a given C library across multiple platforms, its interface must be constant across platforms. DynPort does not support the conditional statements of the $C$ preprocessor. Thus interfaces that use different types for arguments or structure fields depending on the architecture cannot be supported in a universal manner. For example, the Objective-C Run-Time C library of Mac OS $X$ uses a different number of fields within certain struct data types depending on whether the architecture is $i 386$ or alternative AMD64 in which case padding fields are inserted in the middle of the structure. We are aware of this problem although we have not encountered a conflict with the given palette of C libraries available via DynPorts to R. A possible work around for such cases would be to offer separate DynPorts for different architectures.

dyncall and dyncallback currently lack support for handling long double, struct and union argument and return value types and architecturespecific vector types. Work is in progress to overcome this limitation. The middleware BridJ (Chafik,
2011) for the Java VM and C/C++ libraries, which uses dyncall, provides support for passing struct value types for a number of i386 and AMD64 platforms.

$R$ character strings have a maximum size that can limit the number of library functions per dynbind function call. An improved DynPort file format and parser are being developed and are already available for luadyncall.

This version of DynPort does not capture the full range of the $C$ type system. For example array and bit-field types are not supported; the pointer-tofunction type in an argument list can only be specified using the void pointer ' ${ }^{*} \mathrm{~V}$ ' or ' $\mathrm{p}$ ' instead of this (more informative) explicit type. An extended version of DynPort, that overcomes these inconveniences and that improves type safety, is being developed.

Certain restrictions apply when rdyncall is used to work with $C$ libraries. These arise from limitations in R. For example the handling of $\mathrm{C}$ float pointers/arrays and char pointer-to-pointer types are not implemented in $\mathrm{R}$. The functions . unpack and .pack are powerful helper functions designed to overcome these and some other restrictions. Additional helper functions are included, such as floatraw and floatraw2numeric that translate numeric $R$ vectors to $\mathrm{C}$ float arrays and vice versa.

The portable loading of shared libraries via dynfind might require fine-tuning the list of short names when using less common $\mathrm{R}$ platforms such as BSDs and Solaris.

\section{Related work}

Several dynamic languages offer a flexible FFI, e.g. ctypes (Heller, 2011) for Python, alien (Mascarenhas, 2009) for Lua, Rffi (Temple Lang, 2011) for R, CFFI (Bielman, 2010) for Common LISP and the FFI module for Perl (Moore et al., 2008) and Ruby (Meissner, 2011). These all facilitate similar services such as foreign function calls and handling of foreign data. With the exception of Rffi, these also support wrapping of scripting functions as $\mathrm{C}$ callbacks. In most cases, the type information is specified in the grammar of the dynamic language. An exception to this is the Perl FFI that uses text-based type signatures similar to rdyncall.

ctypeslib (Kloss, 2008) is an extension to ctypes that comes closest to the idea of DynPorts in which Python ctypes statements are automatically generated from C library header files, also using GCCXML. In contrast, the DynPort framework contributes a compact text-based type information format that is also used as the main user-interface for various tasks in rdyncall. This software design is applicable across languages and thus type information can be shared across platforms and languages at the 
same time.

Specific alternatives to dyncall include libffi (Green, 2011) and ffcall (Haible, 2004). These are mature FFI libraries that use a data-driven $\mathrm{C}$ interface and have support for many platforms. Although not as popular as the first two, the C/Invoke library (Weisser, 2007) also offers a similar service with bindings to Lua, Java and Kite. The dyncall library offers a functional $\mathrm{C}$ interface (inspired by the OpenGL API). It includes a comprehensive test suite and detailed documentation of calling conventions on a variety of platforms and compilers. As the framework was developed "de novo" we were free to introduce our own strategy to support open as well as commercial and embedded platforms. For example, the i386 Assembly (except for Plan9) is implemented in a common abstract syntax that translates to GNU and Microsoft Assembler. This makes sense here, because i386-based operating systems use a common C calling convention which we address using a single Assembly source. A by-product of this feature is that dyncall enables the user to call operating system foreign code on some architectures.

In contrast to the dynamic zero-compilation approach of ctypeslib and rdyncall, the majority of language bindings to libraries use a compiled approach in which code (handwritten or auto-generated) is compiled for each platform. SWIG (Beazley, 2003) is a development tool for the automatic generation of language bindings. The user specifies the interface for a particular library in a C-like language and then chooses among the several supported languages (including $R$ ) to generate $C$ sources that implement the binding for that particular library/language combination. RGtk2 (Lawrence and Temple Lang, 2011) offers R bindings for the GTK+ GUI framework consisting of $R$ and $C$ code. These are produced by a custom code generator to offer carefully conceived mappings to the object-oriented GObject framework. The generated code includes features such as ownership management of returned objects using human annotations. While custom bindings offer the ability to take into account the features of a particular library and framework to offer very user-friendly mapping schemes, rdyncall aims to offer convenient access to $C$ libraries in general but it requires users to know the details of the particular interface of a $\mathrm{C}$ library and the $\mathrm{R}$ run-time environment.

\section{Summary and Outlook}

This paper introduces the rdyncall package ${ }^{5}$ that contributes an improved Foreign Function Interface for R. The FFI facilitates direct invocation of foreign functions without the need to compile wrappers in C. The FFI offers a dynamic cross-platform linkage framework to wrap and access whole $\mathrm{C}$ inter-

\footnotetext{
${ }^{5}$ Version 0.7.4 on CRAN as of this writing.
}

faces of native libraries from R. Instead of compiling bindings for every library/language combination, $\mathrm{R}$ bindings of a library are created dynamically at run-time in a data-driven manner via DynPort files, a cross-platform universal type information format. $\mathrm{C}$ libraries are made accessible in $\mathrm{R}$ as though they were extension packages and the $\mathrm{R}$ interface looks very similar to that of $C$. This enables system-level programming in $R$ and brings a new wave of possibilities to $\mathrm{R}$ developers such as direct access to OpenGL across platforms as illustrated in the example. An initial repository of DynPorts for standard cross-platform portable $\mathrm{C}$ libraries comes with the package. Work is in progress for implementation of callback support on architectures already supported by the dyncall $\mathrm{C}$ library. The handling of foreign data types, which is currently implemented in $R$ and $C$, is planned to be reimplemented as a $\mathrm{C}$ library and part of the DynCall project.

The DynPort facility in rdyncall consitutes an initial step in building up an infrastructure between scripting languages and $C$ libraries. Analogous to the way in which $R$ users enjoy quick access to the large pool of $\mathrm{R}$ software managed by CRAN, we envision an archive network in which C library developers can distribute their work across languages, users could then get quick access to the pool of $C$ libraries from within scripting languages via automatic installation of precompiled components and using universal type information for cross-platform and cross-language dynamic bindings.

\section{Bibliography}

D. Adler and T. Philipp. DynCall project. URL http://dyncall.org, November 2011. C library version 0.7 .

D. M. Beazley. Automated scientific software scripting with SWIG. Future Gener. Comput. Syst., 19: 599-609, July 2003. ISSN 0167-739X. doi: 10.1016/ S0167-739X(02)00171-1. URL http://portal.acm. org $/$ citation. $\mathrm{cfm}$ ? id $=860016.860018$.

F. Bellard. QEMU, a fast and portable dynamic translator. In USENIX Annual Technical Conference, FREENIX Track, pages 41-46. USENIX, 2005. URL http://www.usenix.org/events/usenix05/ tech/freenix/bellard.html.

J. Bielman. CFFI - common foreign function interface. URL http://common-lisp. net/project/ cffi/, August 2010. CL library version 0.10.6.

O. Chafik. BridJ - Let Java \& Scala call C, C++, Objective-C, C\#... URL http://code.google.com/ $\mathrm{p} / \mathrm{bridj} /$, Jun 2011. Java package version 0.5. 
J. Clark. XSL transformations (XSLT) version 1.1. W3C working draft, W3C, Aug. 2001. URL http: //www.w3.org/TR/2001/wD-xslt11-20010824/.

J. Clark. The Expat XML parser. URL http: / / expat . sourceforge.net/, June 2007. C library version 2.0.1.

D. Flanagan and Y. Matsumoto. The Ruby Programming Language. O’Reilly, Cambridge, 2008.

A. Gavare. GXEmul: a framework for full-system computer architecture emulation. URL http:// gxemul.sourceforge.net/, February 2010. Program version 0.6.0.

A. Green. libffi - a portable foreign function interface library. URL http://sourceware.org/libffi/, August 2011. C library version 3.0.10.

B. Haible. ffcall - foreign function call libraries. URL http: //www.gnu.org/s/libffcall/, June 2004. C library version 1.10 .

T. Heller. ctypes - A foreign function library for Python. URL http://starship.python.net/ crew/theller/ctypes/, October 2011.

R. Ierusalimschy, L. H. de Figueiredo, and W. C. Filho. Lua - an extensible extension language. Software - Practice and Experience, 26(6):635-652, June 1996.

H. Kaiser. Wave V2.0 - Boost C++ Libraries. URL http://www.boost.org/doc/libs/release/ libs/wave/index.html, July 2011. Boost C++ Library Version 1.45, Wave C++ Library Version 2.1.0

E. E. Kim. A triumph of simplicity: James Clark on markup languages and XML. Dr. Dobb's Journal of Software Tools, 26(7):56, 58-60, July 2001. ISSN 1044-789X. URL http://www.ddj.com/.

B. King. GCC-XML. URL http://www.gccxml.org, February 2004. Program version 0.6.0.

G. K. Kloss. Automatic C library wrapping - ctypes from the trenches. The Python Papers, 3(3), 2008. ISSN 1834-3147.

S. Lantinga. libSDL: Simple DirectMedia layer. URL http://www. libsdl.org/, October 2009. C library version 1.2.14

M. Lawrence and D. Temple Lang. RGtk2: A graphical user interface toolkit for R. Journal of Statistical Software, 2011. ISSN 15487660. URL http: //www. jstatsoft.org/v37/i08/paper.
F. Mascarenhas. Alien - pure Lua extensions. URL http: //alien. luaforge.net/, October 2009. Lua module version 0.5.1.

W. Meissner. Ruby-FFI. URL https://github.com/ ffi/ffi/wiki, October 2011. Ruby package version 1.0.10.

P. Moore, G. Yahas, and A. Vorobey. FFI - Perl foreign function interface. URL http: // search.cpan.org/ $\sim$ gaal/FFI/FFI.pm, September 2008. Perl module version 1.04 .

OpenGL Architecture Review Board, D. Shreiner, M. Woo, J. Neider, and T. Davis. OpenGL(R) Programming Guide: The Official Guide to Learning Open $G L(R)$, Version 2. Addison Wesley, 2005.

B. Pendleton. Game programming with the Simple DirectMedia Layer (SDL). Linux Journal, 110:42, 44, 46, 48, June 2003. ISSN 1075-3583.

T. Philipp. DynOS Project. URL http://dyncall. org/dynos, May 2011.

$\mathrm{R}$ Development Core Team. Writing $R$ Extesions. R Foundation for Statistical Computing, Vienna, Austria, 2010. URL http://www.R-project.org. ISBN 3-900051-11-9.

D. Temple Lang. Rffi for run-time invocation of arbitrary compiled routines from R. URL http: //www . omegahat .org/Rffi/, January 2011. R package version $0.3-0$.

L. Tierney. A simple implementation of name spaces for R. URL http://www. stat.uiowa.edu/ luke/ R/namespaces/morenames.pdf, May 2003.

G. van Rossum and F. L. Drake, Jr. Python Language Reference Manual. Network Theory Ltd., 2005. ISBN 0-9541617-8-5. URL http://www. network-theory.co.uk/python/language/.

D. Veillard and B. Reese. The XSLT C library for GNOME. URL http://xmlsoft.org/XSLT/, September 2009. C library version 1.1.26.

W. Weisser. C/Invoke - version 1.0 - easily call C from any language. URL http: / / cinvoke.teegra.net/ index. html, January 2007. C library version 1.0.

\section{Daniel Adler}

Georg-August Universität

Institute for Statistics and Economics

Platz der Göttinger Sieben 5

37079 Göttingen, Germany

dadler@uni-goettingen. de 